\title{
When People are Problematically Attached to Social Media: How Would the Design Matter?
}

\author{
Majid Altuwairiqi, Emily Arden-Close, Elvira Bolat, Laura Renshaw-Vuillier, Raian Ali \\ Bournemouth University, UK \\ \{maltuwairiqi, eardenclose, ebolat, lrenshawvuillier, rali\}@bournemouth.ac.uk
}

\begin{abstract}
Social technology, in particular, social media has increasingly become part of individual's life. People rely on social media interaction to relate with other people and satisfy their leisure or behavioural support purposes. Features such as those presented in the Honeycomb ecology, e.g. sharing, profiling and reputation, are used as means to that end. Recent designs of technology embed social features in them, enabling people to talk to the machine and interact with it as a sentient being, e.g. chatbot and robots, or use it as a mediator for social interaction, e.g. Social Internet of Things (SIoT). However, we argue that over-reliance on social media may result in a problematic attachment to it leading to a negative impact on users' wellbeing. We conduct a multi-stages study to explore the negative psychological states that social media features can trigger. We mainly employ diary studies trying to increase the ecological validity of our data. We also discuss the enrichment of social media with tools to aid people in staying in control of their attachment and the risks such tools can introduce to user experience as well as their behaviour change process.
\end{abstract}

\section{INTRODUCTION}

People worldwide, particularly young people, are embracing social media, which has become deeply interwoven in their daily lives [1]. There is a range of social media platforms and their popularity has increased exponentially over time as a means of facilitating both communication and entertainment [2]. The number of active Facebook users stood at 500 million in 2010 but had increased to 2 billion by 2018 [3]. This rapid expansion has prompted academics to examine its impact on the population with recent studies [4-6] advocating that social media can have both positive and negative impacts on users.

Studies that highlight the negative impacts of social media quite often discuss the term 'social media addiction'. Hawi and Samaha [7] defined social media addiction as compulsive use of social media, resulting in addictive behavioural symptoms. The author in [8] noted six aspects for addictive behavioural symptoms which include "convenience, tolerance, conflict, withdrawal, relapse, and mood modification". Although digital media addiction is currently not classified as a mental disorder in the 5th edition of the Diagnostic and Statistical Manual of Mental Disorder (DSM) [9], given its documented negative impact on wellbeing, further research is deemed much needed.

We recognise that addiction is the possible outcome, but in this paper, we are focusing on a wider than addiction behaviour - attachment to social media that quite often results in the overuse of social media. Attachment theory [10] holds that an attachment style develops based on the interaction between the child and the primary caregiver. Using such a theory, we can define problematic attachment to social media as an over-reliance on social media to satisfy social and personal requirements, which can eventually lead to harms on personal and social wellbeing. People rely on social media to satisfy their attachment needs such as enhance their self-esteem [11] and maximise their social capital [12]. You might question why this is bad for us. However, such reliance can be problematic and associated with harm and side effects on people's well-being such as impaired decision making, e.g. posting pictures under peer pressure, poor impulse control and anxiety leading to checking social media improperly, e.g. while driving [13].

It is evidenced by previous research [11, 12, 14] that people with low self-esteem tend to overuse social media in an attempt to enhance their self-image and self-identity, which positively promotes their self-esteem. However, peers' interaction and failure in getting recognition may worsen their mood. In turn, social media overuse is often linked to negative psychological experiences, including anxiety, depression and stress. It can also contribute to poor academic performance and lead to dissatisfaction with life [7, 15, 16]. In addition, people who develop excessive and obsessive engagement with social media are more likely to experience undesirable life experiences as demonstrated by reduced creativity, increased anxiety and withdrawal from reality [17].

The most critical argument we want to highlight in this paper is that social features embedded within social media technology are not only contributing to social connectivity with others but also to enabling the emotional attachment of social media users with the technology. Such emotional attachment has been shown to be an indicator of intention to use social media and technology. For example, Suh et al. [18] found that those who identified themselves with their Facebook profile picture (avatar) were more emotionally attached to their avatar and had a greater desire to use it. Li et al. [19] found that an individual's emotional connection to an e-commerce website increased the likelihood of them continuing to use that website.

Reflecting on recent development in technology, Reeves and Nass [20] demonstrated that people behave socially towards artefacts like computers and that socially designed robots would intensify this effect. For example, Marti et al. [21] studied the emotional interaction of the seal robot Paro in therapeutic contexts and discovered it had a distinct role in mediating social exchange and stimulating attachment and engagement. 
Moreover, social features are being added to devices and machines creating a new ecology of Social Internet of Things (SIoT) [22] and such an evolution can make the problematic attachment even cyber-physical.

As discussed previously, existing literature on social media addiction has mainly focused on psychological perspectives, such as the role of social skills [23] or personality traits [24], in developing an addiction. However, there is a lack of research in investigating the problematic attachment to social media technology by investigating the role of social media design in both triggering problematic usage and helping to regulate it.

As of 2018, the leading technology companies have released applications for managing addictive digital media behaviours. For example, Apple recently issued the Downtime feature on their iOS system, which helps users monitor the time they spend on digital media, and control notifications. However, it is questionable whether this feature would work beyond enhancing individuals' awareness of their usage. Alrobai and colleagues [25] identified a range of risks in software-assisted behavioural change tools, making their engineering a complex problem and requiring a multidisciplinary approach consisting of expertise from information systems and psychology. This call for further research deepen understanding of the role such software can play in combatting problematic attachment to social media.

In order to know how to design software-assistance to combat problematic social media attachment, an in-depth understanding of the phenomena in a naturalistic, or close to naturalistic setting, is needed. We try to explore such lived experience of the problematic attachment to social media in this research. In particular, we aimed to gain insights into the real-world experience of people who have a problematic attachment to social media. We adopted a multi-stage qualitative research method employing diary studies designed as a primary data collection technique to enhance the ecological validity of the study via using a diary study in a naturalistic setting. We report on the findings regarding the psychological states experienced by the participants. The paper is aimed at informing better use of social media to maintain wellbeing and to drive research-informed development of behavioural change tools to facilitate responsible social media usage.

\section{RESEARCH METHOD}

To obtain more insights into problematic experiences on social media, we conducted a multi-phase qualitative study consisting of an exploratory phase described in Section A and a confirmatory and refinement phase described in Section B. Different qualitative methods were employed in each phase to make sure the data capture and analysis were accurate. The data analysis and framework used as theoretical underpinning are described in Section C. The conceptual diagram of our research methods is presented in Fig.1.

\section{A. First Phase: Exploring Problematic Attachment}

This phase aimed to explore people's problematic experience and attachment to social media. A qualitative method was adopted, which involved focus groups and diary study methods; diary study was the core method in this stage. A focus group was first used to gather initial insights that

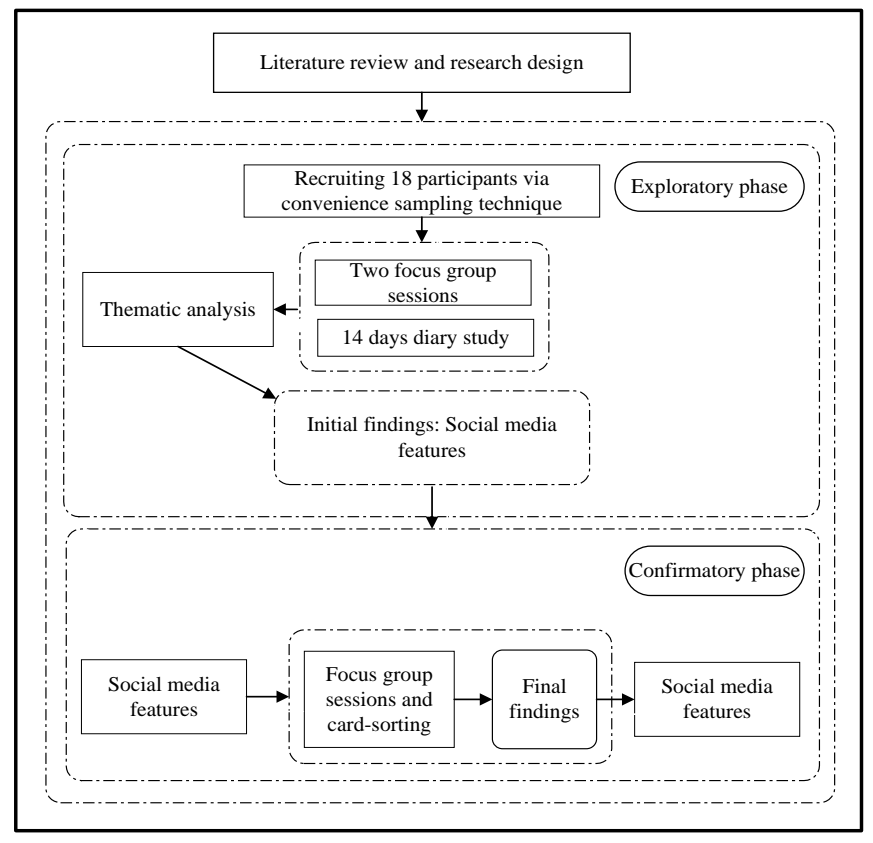

Figure 1. Overview of the research method

were then elaborated via a diary study allowing the capture and refinement of users' problematic experience as lived.

Considering the representative population on social media and the high penetration rate in universities, participants were recruited using convenience sampling in this phase. Eighteen participants aged 18 and over, nine males and nine females, were recruited. Inclusion criteria were being adult social media users and self-identifying as having a problematic attachment to social media.

The inclusion criteria were adhered to using a preselection questionnaire adapted from the Generalized Problematic Internet Use Scale with necessary adaptations [26]. For example, "Internet" was replaced with "social media" to reflect the context.

We conducted two focus groups with nine participants each. The focus group study aimed to help participants familiarise themselves with the study objectives and obtain insights into their problematic relationship and attachment with and on social media. To prepare participants for the diary study in this phase, at the end of each focus group session, specific training on using the Evernote application (app) was provided. Evernote was chosen as it allows users to take notes, pictures, and recordings and share them with the research team daily. The collaborative feature of the Evernote app allows the facilitator to send questionnaires to participants and the research team. The diary study with the same eighteen participants lasted two weeks and involved two tasks. The first focused on identifying their online experience and online behaviour with an emphasis on social media profiling features and online presence. In the second task, participants were asked to detach themselves from a specific activity on social media, such as avoiding an instant reply to their friends' messages as much as possible, to discover accompanying side-effects. The participants provided their notes three times a day (morning, afternoon and night) using the Evernote app. Daily reminders were also sent through the Evernote app. Once the diary study was 
completed, participants were invited for face to face interviews to go through their daily entries.

\section{B. Second Phase: Refinement and Confirmation}

This phase focused on refining the findings of the first phase analysis regarding psychological states and their relation to social media features. A closed card sorting technique within focus group sessions was carried out with the participants.

Fourteen participants were recruited. Six were selected from the first phase sample to avoid analysis bias and increase the credibility of the findings from participants' perspectives. This has served also as a technique for member checking [27], which is used to validate qualitative study findings. The other eight participants were recruited separately following the same recruitment procedure as in Phase 1.

A closed card sorting technique was utilised to refine the first phase findings. The card sorting technique is useful for 1) gaining participants perspective regarding findings and their inter-relations and 2) obtaining additional feedback through discussion and observation. Participants were given the pre-defined primary groups, pens, notes and cards. Cards were related to every category index concluded from the analysis of the first phase, for instance, identity index. The first card sorting session comprised eight participants. The group was split into two teams who had to discuss and sort cards, then discuss them with the other team. In the other session, one-to-one meetings with the participants were held to obtain their individual views. This, in turn, enhanced the process of validation and avoided group thinking [28]. Participants were told to provide the cards and refine the groups and concepts of every category from the initial findings together with the pre-defined cards. Participants were encouraged to "think aloud" as they carried out the card sorting, describing their reasons concerning the grouping and any possible uncertainties which they might encounter. Upon the completion, participants were required to provide clarifications to some questions pertinent to their experience with the sorting exercise.

\section{Data Analysis}

The data collected from the interviews and the diary study was analysed using thematic analysis [29]. In addition, the Honeycomb framework for Social Media Features Ecology [30] was utilised as a starting point to classify the features resulting from the study. It is based on seven feature blocks: identity, conversation, sharing, presence, group, reputation and relationship. However, psychological states toward social media features were the main findings of the analysis process. The published literature in problem gaming and substance abuse [8, 31] reinforced our findings in problematic behaviours. In light of this background, the nature of the social media platform was taken into account. The findings were also validated through the card sorting in the second phase of the study.

\section{FINDINGS: Social MEdia FEATURES Vs PSYCHOLOGICAL STATES}

The design features attributed to social media can play a crucial role in promoting problematic attachment and subsequent side-effects. Engineers are faced with challenges of the practical introduction of social media design as well as managing their influences. Side-effects such as anxiety might accompany the ad-hoc change of social media features. Adhoc changes, such as limited access to some features for individuals who maintain a high level of digital attachment, may contribute to negative psychological states as a result of the user's loss of popularity.

The Honeycomb framework [30] was used as a template analysis to help classify the features of social media resulting from the exploratory study (Phase I, Section A). The purpose was to investigate the role of the features of social media, as classified by the Honeycomb framework, in facilitating certain psychological states and problematic attachment patterns. Features such as push notifications and direct communications were added to the seven main features of the Honeycomb framework to meet the requirements of the study and the findings of the analysis. A detailed explanation of the design features derived from the analysis is provided below. Most features from the Honeycomb framework contribute to one or more than one psychological states as presented in Fig.2.

1) Identity: Identity is the feature that is required for individuals to set up their account and reveal their identities on social media. Identity feature includes the profile information personal details such as name and picture and the profile content such as the timeline and posts dashboard. When individuals identify themselves they can disclose aspects of their personal information, including their name, age, gender, status, interests, location, background, and

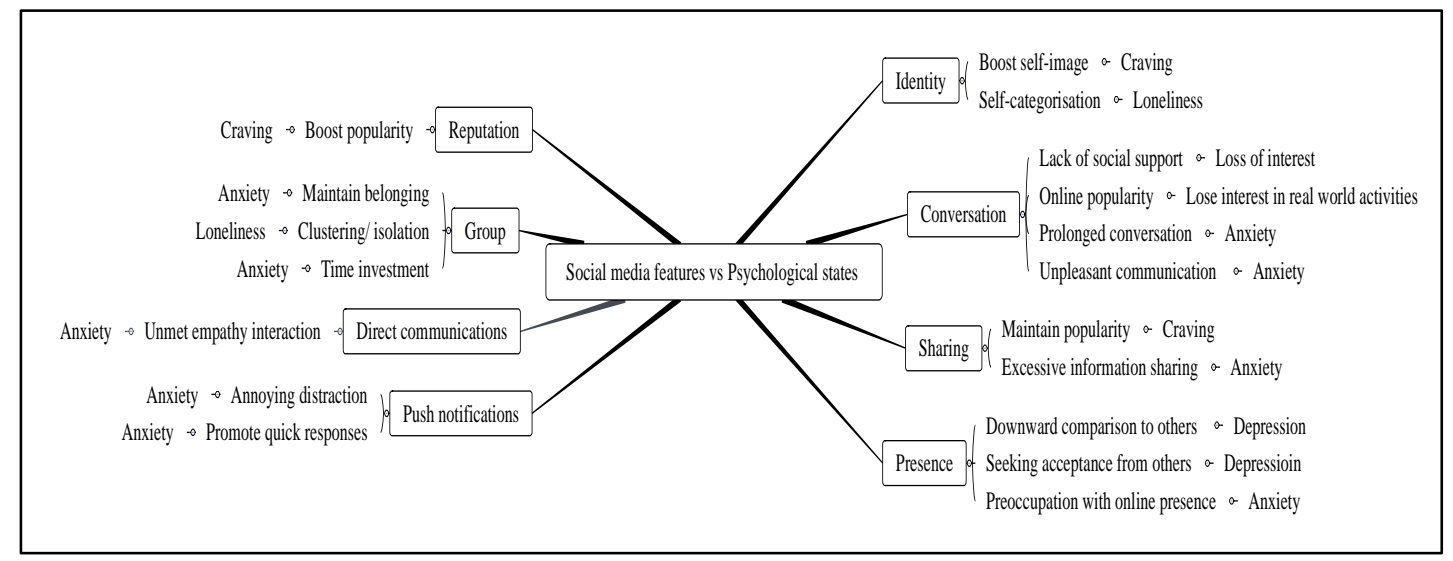

Figure 2. Problematic attachment to Social Media: Social Media Features Vs Psychological States 
profile picture. The details and level of disclosure of real information are different from one user to another depending on their personality and their reason for using the social media site. For example, the identity could be real with a real name, picture and profile information or it could be anonymous without enough information leading to the real identity. Alternatively, it could be between the two. There is also an implicit disclosure of their online identity through their online interaction style such as a quick response when they receive notifications, or through profile content which is present in the timeline, as it is on Facebook. This is an indirect disclosure of online identity and interests. This feature could facilitate craving and loneliness in relation to a problematic attachment to social media.

Our results show that the identity feature could facilitate craving when individuals interact on social media to maintain their self-concept. However, the identity feature could also be beneficial in reinforcing individual or group identity. Having an identity gives the individual a sense of belonging, which in turn enhances their self-concept and self-esteem. Thus, individuals with a problematic attachment may crave online interaction to boost their self-image. They may feel a craving to create a community and place of belonging, relying heavily on the identity feature to search for similarly identified profiles to interact with: "I rely on profile information to find people who share the same characteristics such as gender, age and workplace".

Our results also show that online identity could facilitate loneliness. Individuals can express their self through profile information and content. This information leads individuals comparing their profiles to others and self-categorising, which in turn lowers their self-esteem and provokes loneliness: "In my opinion, journalists are well educated. I feel lonely in social media because I only make contact with people from the same field".

2) Conversation: The conversation feature refers to communication that occurs between social media users via features such as posts, comments, likes, chat, and sharing. Communicating with other users or groups is the primary reason for some individuals to use social media. The conversations can be used to share opinions with likeminded individuals, promote oneself, boost self-esteem, and keep up to date with the latest knowledge. Social media can also provide a secure means of communicating with friends and family. Crucially, if an individual feels that the system is secure, they are likely to be more open and perhaps build relationships. However, this online interaction can lead some users to be more attached to social media and facilitate problematic usage, resulting in anxiety and a loss of interest.

In our research, we found that individuals on social media rely on the conversation feature to communicate with others because it is instantaneous and easy to access. This can result in a problematic attachment and a loss of interest if online friends are unavailable for social support. Additionally, it could facilitate a problematic attachment to social media and drive individuals to communicate online rather than face to face, thus allowing them to boost their online popularity and relatedness in a way which is unproportioned to real-life contacts. Consequently, individuals may lose their interest in and enjoyment of activities in the real world: "I like to have online conversations with others which make me feel happier than if I did not do anything".

While considering such imbalance as a problem can be debated, we note here that the participants in our study selfdeclared considering their relation to social media as problematic in the pre-selection criteria.

We also found that the conversation feature promotes a problematic attachment to social media by enabling individuals to engage in online conversations excessively. Individuals are communicating with others and losing awareness of the time spent online by maintaining these relationships. Consequently, individuals could feel anxious about prolonged conversation: "I rely on online conversation to keep my relationship, but I feel anxious because it is difficult to ignore any conversation".

In addition, we found that individuals may engage in online conversation excessively to explore others, leading to a problematic attachment. Thus, they may find it challenging to keep a distance from unpleasant communication or content, which in turn triggers a feeling of anxiety. For example, in the real world individuals can maintain distance between themselves and others through avoiding being geographically close. This can be difficult in the online space especially for people who overly rely on it for meeting their social needs.

3) Sharing: The sharing feature is characterised by exchanging and distributing social media content between users. It is a fundamental feature on social media platforms such as Flicker, Facebook, Twitter, and YouTube. For individuals with a problematic online attachment, the sharing feature can facilitate psychological states such as craving and anxiety.

Our research found that users feel the desire to increase their popularity and maintain it in social media, which has a positive impact on their self-esteem. The sharing of posts, videos, and content with friends and groups assist them in increasing their popularity. Therefore, individuals may develop problematic attachment because they rely on the sharing feature to maintain popularity and sense of belonging, which may eventually promote a sense of craving: "I have a good reputation in social media because of my unique stories. I crave to share any news as soon as it is received. Sharing is a powerful technique in social media".

Our findings highlight that sharing is an important social activity. Many individuals share information on social media to gain acceptance from others. Sharing behaviour at this stage, facilitated by the design of social media, e.g. promoting certain sharing's according to the interest, can facilitate problematic attachment and raise anxiety due to excessive information sharing: "I worry about sharing too 
much information in social media; I cannot stop myself from doing this".

In addition, individuals may feel anxious regarding ambiguous or unpleasant content shared by others. This happens, for example, when users label their friends using hashtags or name them in a picture while friends are not interested in the content and its context.

4) Presence: The presence feature is concerned with how users can determine whether or not other individuals can be contacted or available. This could be as simple as being aware of another person's status and whether they are contactable (e.g. away, busy, hidden, and available). The status line on social media offers another way of signalling whether or not an individual is available, e.g. writing in the status line the availability time. An alternative sign of an individual's presence is their degree of engagement via social media. For instance, if they regularly post and chat then this will have the effect of signalling to others that they are likely to be present at any given time. Another example is the 'last seen' feature in some programs such as WhatsApp and Skype. This subsequently provokes a sense of closeness to others. In terms of group dynamics, presence is indicative of cohesion among a group. In addition, presence can be associated with self-presentation which is associated with facilitating problematic attachment. Therefore, the presence feature can facilitate a problematic attachment which consequently triggers psychological states such as depression and anxiety.

In our research, we found that individuals who tend to engage in a lot of social media activities may feel that they do not live up to the lifestyles that others tend to present in their profiles. Such a peer comparison facilitates problematic attachment to social media in which some would try to imitate or compete with peers' online profile. Therefore, this kind of downward comparison leads to them feeling occasionally depressed. In addition, presence in social media can influence an individual's behaviour. Thus, individuals develop a problematic attachment and rely on their online presence to seek acceptance from others. They are greatly influenced by the behaviour of others or by group norms, which leads them to feel a degree of depression: "I am a member of more than one group in Facebook, and there are a set of norms for each group to maintain each participants, presence which leads me to interact differently in each group, which make me feel frustrated".

Moreover, they may feel depressed if they are searching online for their friends, but no one is available to communicate and when they find no updates on their status: "One activity I enjoy in social media to escape from the real life is communicating with my friends online and checking their status updates. I feel depressed if I could not find one of them online".

Additionally, the presence feature could facilitate a problematic attachment and trigger anxiety towards preoccupation with online presence to maintain relatedness and proximity. For example, individuals who engage in social media and overly rely on their presence and availability are preoccupied with the latest update in social media or being available at the right time to interact with others, which in turn promote anxiety feeling.

5) Push notifications: It is conceptually an event-based mechanism where senders or social media platforms push events and information to others to notify them about what has happened. There are different ways to receive notifications, such as status updates, vibration, voice, email, message, or groups of notifications to notify people that they have received a notification. This is a reinforcement feature and has the indirect implication of triggering people to interact through social media. Therefore, push notification facilitates psychological states in relation to problematic attachment to social media. This can be due to being interrupted while working or resistance to interact [32].

Push notifications attract people's attention by notifying them concerning online activity. People could receive number of notifications over the day from their social media profiles. We found that the overload of notifications could facilitate problematic attachment to online interaction due to annoying distraction and interrupting tasks, which in turn can provoke anxiety: "I get a lot of alerts through WhatsApp and Snapchat and urges me to respond and communicate with others excessively, which makes me anxious".

In addition, notifications could lead people to promote quick responses, thereby creating hasty behaviour and problematic attachment to social media through a daily routine. Thus, people may feel anxious when they receive notifications, leading them responding quickly: "I feel anxious about notifications. I cannot stop myself from responding to them when they reach me". The low impulse control seems to be a common characteristic in such behaviour.

6) Direct communication: This refers to direct communications between users by means of comments, likes and posts in order to remain connected, return a sense of belonging and increase popularity [33]. A lot of effort is needed to maintain these advantages. Our research discovered that such effort could result in problematic attachment to social media which in turn could trigger psychological states such as anxiety when posts are not responded to especially be reciprocation is expected: "I feel anxious if I have not received likes and positive comments on my daily posts compared to the time I invested in others' posts".

7) Group: Group refers to the ability of users to create communities or sub-communities in social media in order to share their experiences with others. Groups will usually have a specific identity and the group's members will usually share common characteristics in terms of age, family relations, gender and opinion. From the exploratory study, it was clear that the group feature facilitates psychological states in relation to problematic attachment to social media.

Once a user has engaged in a group, they start interacting with the group members to achieve affiliation. We found 
that in order to develop this affiliation, they will make a greater effort to post and share contents to receive attention from others in the group. This may facilitate the development of problematic attachment to social media. This, in turn, may trigger anxiety towards time investment in these groups or from the effort and excessive use to maintain belonging: "The use of social media, especially groups, makes me anxious about investing too much time interacting with others to make a good impression".

On the other hand, all group members have a common identity with norms and characteristics which distinguish them from other groups. Thus, this may lead them to cluster themselves and consequently trigger social isolation. In case of loss of membership from the group or inability to access it, individuals may feel lonely because there became overly reliant on it without growing alternative options to feel connected: "I feel loner if I could not access my online group, I feel my group we name it [anonymized for confidentiality reasons] is my comfort zone".

8) Reputation: The reputation feature in social media is the extent to which users can determine the popularity of others, including themselves. Reputation can have different meanings on social media platforms. In most cases, reputation is a matter of trust. However, we found that reputation in social media does not only refer to identity, but also refers to the content they provide on their profile, which is often evaluated by others. For example, on Twitter, people re-tweet or like content, which in turn adds value to the reputation. In addition, our results show that the number of followers on social media determines how popular a person is, since people can follow as many others as possible. Thus, reputation status can be determined by the number of views, followers, or through evaluation system such as hashtag, which enables individuals to monitor the spread of his content. The reputation feature could facilitate problematic attachment if we take into account that people like to be popular which could positively affect their self-esteem. Thus, this feature may trigger psychological states such as craving accompanied with sacrificing other requirements such as privacy: "I strive to be one of the famous in Snapchat; I share every moment of my life with others".

\section{Discussions: PROBLEMATIC ATtACHMENT AND} BeHAVIORAL CHANGE TOOLS IN SOCIAL MEDIA

Our research confirmed that people experience psychological states including anxiety, depression and lower well-being in combination with their social media usage. While we are not advocating a causal relation between social media usage and such states, one of the possible triggers relates to the design features. Social media is originally meant to provide social support, return a sense of connectedness among users and promote social capital and self-esteem. Some design features seem to rely on gratification through positive expectations, social recognition, feedback and rewards to persuade online interaction and increase usage.
Despite the positive outcomes of online interaction, it may be linked to negative impacts on well-being [34]. Ultimately, a design that promotes positive wellbeing can support users values, e.g. social satisfaction and system trustworthiness and trust. The design could play an essential role in promoting and helping healthy online interaction. For example, current social media design allows users to accept or reject friend requests and set up privacy settings which enhance trust in the system.

Users with problematic attachment are characterised by denial of their online behaviour, reactance when they feel their freedom to continue using social media is controlled by some measures like reminders and timers, and relapse when they try to regulate that relation both in terms of usage style and preoccupation around it [35-37]. Thus, the design of social media needs to consider the differences that users may have in their characteristics on different configurations of software-based intervention to meet the social and wellbeing needs of users [38]. In addition, users showing problematic attachment to social media may experience cravings to increase their reputation or feel anxiety when unable to connect or interact as wished. In our study, we found a set of negative psychological states which are facilitated by features of social media such as grouping, conversation, relationship and reputation. This suggests that the current design adopted by social media may impact negatively on users' wellbeing with the absence of any countermeasures to help mechanisms like self-regulation and mindfulness. Suggestions have been made that individuals with poorer wellbeing are more likely to use social media in order to alleviate loneliness. However, a recent large-scale longitudinal study found that greater use of Facebook was associated with poorer future wellbeing and that this correlation was stronger than the one between realworld social interaction and positive well-being [39].

Fogg in [40] developed a behaviour model for persuasive design, which holds that in addition to ability and motivation, successful behaviour change requires triggers. These are best described as reminders to engage in a particular behaviour. A spark is a particular type of trigger that also contains a motivational element and is particularly effective in changing behaviour when motivation is low. An example is aspects of social media such as push notifications, which increase motivation to check social media, something that individuals might otherwise have done less frequently. If an individual checks social media while working, or otherwise productively engaged, he/she is likely to experience anxiety due to work time missed. Receiving notifications constantly due to 'likes' on a post feeds social satisfaction, but may at the same time increase anxiety due to time spent checking social media. Further notifications may lead to unplanned checking of social media in order to enhance gratification, which may at the same time contribute to increased distress.

Software is usually designed in line with user requirements. However, in this case, user requirements regarding social interaction and connection may contribute to problematic attachment to social media. Amendments to software design in order to meet care requirements are therefore needed. Software design can be used to aid people with tools that include persuasive and gamification techniques to reform their problematic attachment with social media. However, ad hoc empowering of social media design 
by these tools may itself result in side effects such as anxiety and stress on users which lead to loss of interest to engage in the behaviour change process [41]. We present some of these challenges in Table I.

TABLE I. INNOVATIVE DESIGN AND THEIR CONSIDERATIONS

\begin{tabular}{ll}
\hline \multicolumn{1}{c}{ Innovative design } & \multicolumn{1}{c}{ Considerations } \\
\hline Reminder to decrease use & Preoccupations, cravings \\
Push notification off & Anxiety \\
Reducing access & Loneliness \\
Participatory design to set goals & Denial of issues, setting achievable \\
& and appropriate goals (neither too \\
& difficult nor too easy) \\
\hline
\end{tabular}

Co-creation methods (involving service users in the development of interventions) facilitate the integration of user perspectives with expert opinion and scientific evidence [42]. Participatory design has been used successfully in the development of interventions, such as in improving the dynamics and challenges of serious games for health promotion [43]. Participatory design methods [44] may, therefore, provide a solution to reducing this conflict in order to develop platforms that are acceptable to both users on the one hand, in terms of ease of use and satisfying needs, and developers on the other, in terms of promoting wellbeing, in line with corporate social responsibility.

However, with participatory design methods, there are a number of issues to be taken into consideration. Care needs to be taken when including individuals with problematic behaviour in the design of interventions, to ensure that such interventions provide an appropriate message to the target population. For example, such individuals may be in denial regarding the extent of their issues [25]. They may, therefore, trivialise the seriousness of the problem, suggesting that unusually high levels of social media usage are normal. Setting standards that are too easy, so users receive high levels of positive feedback may lead to some individuals believing they are cured whilst their usage is still problematic [45]. On the other hand, setting unrealistic standards that individuals find it difficult to adhere to may lead to reductions in self-esteem, and consequently increases in depression. Such conflicting requirements need to be managed carefully.

It is also important that design to target problematic attachment to social media does not lead to other issues. Individuals with problematic attachment to social media tend to be anxious [46] and may report that, e.g., checking notifications reduces their anxiety. Identifying designs of social media platforms that simultaneously facilitate improved wellbeing and reduced problematic social media usage is a challenge to be addressed. For example, a design that provided individuals with fewer notifications, while reducing triggered checking, might increase anxiety in individuals who are used to checking social media excessively frequently. Also, limiting groups to certain times, while reducing the pressure to reply to messages at all hours, might lead to increased loneliness in individuals who have come to rely on the group for support due to its 24-7 availability. Such risks suggest that a socio-technical approach is much needed, e.g. a blended approach where human care is joined with machine intelligence and software tools.
Despite these issues, co-design has been successfully implemented with individuals with problematic behaviour. For example, in a recent study, a workbook to address alcohol use when quitting smoking was successfully created with input from individuals who drink alcohol to hazardous levels [47]. It, therefore, has potential as a method for working with individuals with problematic attachment to social media.

\section{CONCLUSION}

In this paper, we explored the problematic attachment to social media presented by users in relation to the design features of social media platforms. Our findings revealed psychological states associated with problematic attachments and how social media design features facilitated these states. Our study provided an ecological exploration through the use of diary entries for data collection. In particular, we identified relations between design features and psychological states associated with their online interaction. Our findings suggest that social media can be equipped with tools to help manage such a problematic attachment and help users to reform their online interaction, expectations and online identity in a healthy manner. The design of these behavioural change tools is complicated due to the characteristics of people with problematic attachment, such as denial, relapse and cognitive dissonance. Most of the literature on the problem domain focused on user psychology and their reason to be attached. Thus, in our future work, we will get more insight into the manifestations of psychological states associated with the problematic attachment through other methods of real-time data such as wrestling sensors in order to promote crosssectional study. This will help better understanding of the topic domain and pave the way for clustering people who experienced problematic attachment to social media in order to customize and empowering countermeasures, e.g. persuasive techniques to fit each group.

\section{REFERENCES}

[1] T. A. Pempek, Y. A. Yermolayeva, and S. L. Calvert, "College students' social networking experiences on Facebook," Journal of Applied Developmental Psychology, vol. 30, pp. 227-238, 2009.

[2] G. S. O'Keeffe and K. Clarke-Pearson, "Clinical report-the impact of social media on children, adolescents, and families," Pediatrics, pp. peds. 2011-0054, 2011.

[3] Facebook. (2019). Facebook Newsroom. Available: https://newsroom.fb.com/company-info/

[4] P. Charoensukmongkol, "The Impact of Social Media on Social Comparison and Envy in Teenagers: The Moderating Role of the Parent Comparing Children and In-group Competition among Friends," Journal of Child and Family Studies, vol. 27, pp. 69-79, 2018.

[5] M. Altuwairiqi, T. Kostoulas, G. Powell, and R. Ali, "Problematic attachment to social media: lived experience and emotions," in World Conference on Information Systems and Technologies (WorldCIST), 2019.

[6] M. Altuwairiqi, E. Arden-Close, N. Jiang, G. Powell, and R. Ali, "Problematic Attachment to Social Media: the Psychological States vs Usage Styles," presented at the 13th International Conference on Research Challenges in Information Science (RCIS), 2019.

[7] N. S. Hawi and M. Samaha, "The relations among social media addiction, self-esteem, and life satisfaction in university students," Social Science Computer Review, vol. 35, pp. 576-586, 2017.

[8] M. Griffiths, "A 'components' model of addiction within a biopsychosocial framework," Journal of Substance Use, vol. 10, pp. 191-197, 2005.

[9] A. P. Association, Diagnostic and statistical manual of mental disorders (DSM-5®): American Psychiatric Pub, 2013. 
[10] J. Bowlby, "Attachment and loss v. 3 (Vol. 1)," Random House. Furman, W., \& Buhrmester, D.(2009). Methods and measures: The network of relationships inventory: Behavioral systems version. International Journal of Behavioral Development, vol. 33, pp. 470$478,1969$.

[11] A. L. Gonzales and J. T. Hancock, "Mirror, mirror on my Facebook wall: Effects of exposure to Facebook on self-esteem," Cyberpsychology, Behavior, and Social Networking, vol. 14, pp. 7983, 2011.

[12] C. Steinfield, N. B. Ellison, and C. Lampe, "Social capital, selfesteem, and use of online social network sites: A longitudinal analysis," Journal of Applied Developmental Psychology, vol. 29, pp. 434-445, 2008.

[13] H. C. Woods and H. Scott, "\# Sleepyteens: social media use in adolescence is associated with poor sleep quality, anxiety, depression and low self-esteem," J Adolesc, vol. 51, pp. 41-49, 2016.

[14] L. Denti, I. Barbopuolos, I. Nilsson, L. Holmberg, M. Thulin, M. Wendeblad, L. Andén, and E. Davidsson, "Sweden's largest Facebook study," 2012.

[15] D. J Kuss, M. D Griffiths, L. Karila, and J. Billieux, "Internet addiction: a systematic review of epidemiological research for the last decade," Current pharmaceutical design, vol. 20, pp. 4026-4052, 2014.

[16] A. Lepp, J. E. Barkley, and A. C. Karpinski, "The relationship between cell phone use, academic performance, anxiety, and satisfaction with life in college students," Computers in Human Behavior, vol. 31, pp. 343-350, 2014.

[17] C. S. Andreassen, "Online social network site addiction: A comprehensive review," Current Addiction Reports, vol. 2, pp. 175184, 2015.

[18] K.-S. Suh, H. Kim, and E. K. Suh, "What if your avatar looks like you? Dual-congruity perspectives for avatar use," MIs Quarterly, pp. 711-729, 2011.

[19] D. Li, G. J. Browne, and J. C. Wetherbe, "Why do internet users stick with a specific web site? A relationship perspective," International journal of electronic commerce, vol. 10, pp. 105-141, 2006.

[20] B. Reeves and C. I. Nass, The media equation: How people treat computers, television, and new media like real people and places: Cambridge university press, 1996.

[21] P. Marti, A. Pollini, A. Rullo, and T. Shibata, "Engaging with artificial pets," in Proceedings of the 2005 annual conference on European association of cognitive ergonomics, 2005, pp. 99-106.

[22] L. Atzori, A. Iera, and G. Morabito, "From" smart objects" to" social objects": The next evolutionary step of the internet of things," IEEE Communications Magazine, vol. 52, pp. 97-105, 2014.

[23] O. Turel and A. Serenko, "The benefits and dangers of enjoyment with social networking websites," European Journal of Information Systems, vol. 21, pp. 512-528, 2012.

[24] A. Winkler, B. Dörsing, W. Rief, Y. Shen, and J. A. Glombiewski, "Treatment of internet addiction: a meta-analysis," Clin Psychol Rev, vol. 33, pp. 317-329, 2013.

[25] A. Alrobai, J. McAlaney, K. Phalp, and R. Ali, "Exploring the risk factors of interactive e-health interventions for digital addiction," in Substance Abuse and Addiction: Breakthroughs in Research and Practice, ed: IGI Global, 2016, pp. 375-390.

[26] S. E. Caplan, "Problematic Internet use and psychosocial well-being: development of a theory-based cognitive-behavioral measurement instrument," Computers in Human Behavior, vol. 18, pp. 553-575, 2002.

[27] L. Birt, S. Scott, D. Cavers, C. Campbell, and F. Walter, "Member checking: a tool to enhance trustworthiness or merely a nod to validation?," Qualitative Health Research, vol. 26, pp. 1802-1811, 2016.

[28] B. L. Berg, H. Lune, and H. Lune, Qualitative research methods for the social sciences vol. 5: Pearson Boston, MA, 2004.

[29] V. Braun and V. Clarke, "Using thematic analysis in psychology," Qualitative Research in Psychology, vol. 3, pp. 77-101, 2006.

[30] J. H. Kietzmann, K. Hermkens, I. P. McCarthy, and B. S. Silvestre, "Social media? Get serious! Understanding the functional building blocks of social media," Business Horizons, vol. 54, pp. 241-251, 2011.

[31] R. Tao, X. Huang, J. Wang, H. Zhang, Y. Zhang, and M. Li, "Proposed diagnostic criteria for internet addiction," Addiction, vol. 105, pp. 556-564, 2010.
[32] M. Pielot and L. Rello, "Productive, anxious, lonely: 24 hours without push notifications," in Proceedings of the 19th International Conference on Human-Computer Interaction with Mobile Devices and Services, 2017, p. 11.

[33] M. Burke and R. Kraut, "Using Facebook after losing a job: Differential benefits of strong and weak ties," in Proceedings of the 2013 conference on Computer supported cooperative work, 2013, pp. 1419-1430.

[34] C. Huang, "Time spent on social network sites and psychological well-being: A meta-analysis," Cyberpsychology, Behavior, and Social Networking, vol. 20, pp. 346-354, 2017.

[35] J. Littrell, "How addiction happens, how change happens, and what social workers need to know to be effective facilitators of change," Journal of evidence-based social work, vol. 8, pp. 469-486, 2011.

[36] J. J. Kandell, "Internet addiction on campus: The vulnerability of college students," CyberPsychology \& Behavior, vol. 1, pp. 11-17, 1998.

[37] P. Ann Stoddard Dare and L. Derigne, "Denial in alcohol and other drug use disorders: A critique of theory," Addiction Research \& Theory, vol. 18, pp. 181-193, 2010.

[38] A. Shahri, M. Hosseini, M. Almaliki, K. Phalp, J. Taylor, and R. Ali, "Engineering software-based motivation: a persona-based approach," in Research Challenges in Information Science (RCIS), 2016 IEEE Tenth International Conference on, 2016, pp. 1-12.

[39] H. B. Shakya and N. A. Christakis, "Association of Facebook use with compromised well-being: a longitudinal study," American journal of epidemiology, vol. 185, pp. 203-211, 2017

[40] B. J. Fogg, "A behavior model for persuasive design," in Proceedings of the 4th international Conference on Persuasive Technology, 2009, p. 40.

[41] G. Drosatos, F. Nalbadis, E. Arden-Close, V. Baines, E. Bolat, L. Vuillier, T. Kostoulas, S. Wasowska, M. Bonello, and J. Palles, "Empowering Responsible Online Gambling by Real-time Persuasive Information Systems," presented at the Research Challenges in Information Science (RCIS), 2018.

[42] D. L. Sackett, W. M. Rosenberg, J. M. Gray, R. B. Haynes, and W. S. Richardson, "Evidence based medicine: what it is and what it isn't," ed: British Medical Journal Publishing Group, 1996.

[43] A. DeSmet, D. Thompson, T. Baranowski, A. Palmeira, M. Verloigne, and I. De Bourdeaudhuij, "Is participatory design associated with the effectiveness of serious digital games for healthy lifestyle promotion? A meta-analysis," Journal of medical Internet research, vol. 18, 2016.

[44] D. Schuler and A. Namioka, Participatory design: Principles and practices: CRC Press, 1993

[45] W. B. Frick, "Flight into health: A new interpretation," Journal of humanistic psychology, vol. 39, pp. 58-81, 1999.

[46] S.-W. Choi, D.-J. Kim, J.-S. Choi, H. Ahn, E.-J. Choi, W.-Y. Song, S. Kim, and H. Youn, "Comparison of risk and protective factors associated with smartphone addiction and Internet addiction," J Behav Addict, vol. 4, pp. 308-314, 2015.

[47] N. Minian, A. Noormohamed, L. Zawertailo, D. Baliunas, N. Giesbrecht, B. Le Foll, J. Rehm, A. Samokhvalov, and P. L. Selby, "A method for co-creation of an evidence-based patient workbook to address alcohol use when quitting smoking in primary care: a case study," Research involvement and engagement, vol. 4, p. 4, 2018. 